\title{
Behavioral augmentation of tolerance to alcohol and the response measure
}

\author{
LOWELL T. CROW and MARK W. HIGBEE \\ Western Washington State College, Bellingham, Washington 98225
}

\begin{abstract}
The differential effects upon behavior of alcohol in tolerance acquisition were examined. Consistent with earlier findings using a treadmill and temporal maze, tolerance as assessed by changes in operant response duration was transferable to another operant task, and tolerance as measured by operant response duration was augmented by drug-related practice only in the rate of tolerance acquisition, not in the level of tolerance attained. However, with the use of response rate as the acquisition index, tolerance was not completely transferable to a different operant and drug-related practice augmented rate of acquisition as well as final level of tolerance attained. It is suggested that the response measure employed is a variable in the determination of the behavioral augmentation of alcohol tolerance in the rat.
\end{abstract}

Although the acquisition of alcohol tolerance in the rat may involve specific metabolic changes resulting in a lowering of blood alcohol level, these changes are probably not large enough to account for the known parameters of response recovery, and some kind of adaptation or desensitization to the central nervous state occasioned by the alcohol has been proposed (Kalant, 1973; Kalant, LeBlanc, \& Gibbins, 1971). The role of learning in such tolerance acquisition is acknowledged as important, but primarily catalytic in nature. In an elaboration of studies by Chen (1967, 1968), LeBlanc, Gibbins, and Kalant (1973) were able to show that tolerance by rats in a temporal circular maze task was acquired more quickly with drug-related practice than with equal amounts of the drug alone, but that the final level of tolerance was not affected by such learning. In addition, it had been shown that the tolerance was transferable from a treadmill situation to that of the temporal circular maze and vice versa (Kalant, 1973).

As previous work by one of the present authors suggested a differential rate of alcohol tolerance acquisition in operant conditioning depending upon the measure employed, response duration or rate (Crow, Westveer, \& Kass, 1976), the present studies were undertaken to examine the questions of task specificity and final tolerance level using these operant behavioral measures.

\section{EXPERIMENT 1}

\section{Method}

Subjects. Sixteen Sprague Dawley albino rats (12 females and 4 males) were used. The animals were approximately 200 days of age at the beginning of training and naive to alcohol and water deprivation.

Apparatus. The basic apparatus consisted of two baractivated water delivery operant boxes and two free-access drinking boxes. The latter two boxes were equipped with GrasonStadler drinkometers and, by means of running-time meters and counters, measures were obtained of number and duration of licks during the 30 -min experimental session. A stopwatch was used to measure intrasession intervals which were $2,4,8$, and 16 min duration (i.e., taken at 2,6,14, and 30 min elapsed time). Each of the four boxes was equipped with two sets of timers and counters to facilitate the ease of reading as alternate sets of recording instruments were used, one being active while the other was idle. A single selector switch controlled this function for all four boxes, so four animals could be run simultaneously.

Procedure. All animals were maintained on a $23 \frac{1}{2}-\mathrm{h}$ waterdeprivation schedule throughout the experiment and run in groups of four. The experiment consisted of three basic phases: Phase 1a (training) involved the training of one half of the animals in barpressing in the operant boxes on a continuous reinforcement (CRF) schedule, and the remaining half in the free-access drinking boxes. Phase $1 \mathrm{~b}$ (training) consisted of training each animal in the other task, that is, training in the operant box for those animals in Phase 1a which were trained in the drinking boxes and training in the drinking boxes for those previously trained in the operant boxes. Phase 2 (alcohol tolerance acquisition) involved alternate-day i.p. ethanol injections for all animals for the tasks assigned in Phase 1b. Injections were given $10 \mathrm{~min}$ prior to the experimental session. For half the animals the dosage was always $.5 \mathrm{~g} / \mathrm{kg}$, and for the other half always $1.0 \mathrm{~g} / \mathrm{kg}$. The injections were continued so that data for a total of six injections for each animal were obtained. The procedure for those days on which no injections were carried out was identical to the procedure in Phase 1b. Phase 3 (task change) consisted of maintaining the procedures of Phase 2 with the sole exception that for each animal the task was changed to that of Phase 1a. That is, identical alcohol treatments on alternate days were continued, but animals in the operant box in Phase 2 were switched to the drinking boxes and those in the free-access drinking boxes in Phase 2 were switched to the operant boxes. Phase 3 was carried out so that data from a total of two alcohol treatment days were obtained.

\section{Results}

Response rate. The data of the number of responses for the 30-min sessions, both barpresses and licks, were pooled by computing for each animal the percentage deficit from one phase of the experiment to another. Thus, the percentage deficits from the last 2 days (mean deficit) of the training period (Phase 1) to the 2 treat- 
ment days of the task change period (Phase 3) were subjected to a 2 by 2 ANOVA of dosage level by task level. Dosage was significant at the $5 \%$ level of confidence $(F=4.89, \mathrm{df}=1 / 12$, but neither the task effect nor the interaction was significant $(F=.84, \mathrm{df}=1 / 12$; $\mathrm{F}=2.82, \mathrm{df}=1 / 12$, respectively). A similar analysis of the percent deficit scores of number of responses from the last tolerance day (Phase 2) yielded no significant dosage effect $(F=1.41, \mathrm{df}=1 / 12)$, no significant task effect $(F=1.25, \mathrm{df}=1 / 12)$, and no significant interaction $(\mathrm{F}=1.04, \mathrm{df}=1 / 12)$.

Figure 1 summarizes these data in standard scores based upon the training data (Phase 1). Note that the data are collapsed across the task variable, and that the alternate days of no treatment during Phases 2 and 3 are presented as a continuation of the training data function.

Response duration. The response duration or microbehavioral data were averaged for each animal for the entire 30-min drinking period, and these data were used to compute percent deficit scores from one experimental treatment to another. Thus, percent deficit scores were derived for all animals from the last 2 training days (Phase 1) to the 2 treatment days of Phase 3 , and a 2 by 2 ANOVA was carried out using the dosage levels and task levels above. There was no significant dosage effect $(F=1.36$, df $=1 / 12)$, no task effect $(F=.64$, $\mathrm{df}=1 / 12)$, and no interaction $(\mathrm{F}=1.92, \mathrm{df}=1 / 12)$.

\section{Discussion}

The results indicate that the acquisition of tolerance to alcohol in terms of a difference in rate of responses between the two dosage levels (.5 and $1 \mathrm{~g} / \mathrm{kg}$ ) occurred at the sixth day of alcohol treatment. That is, using the training (Phase 1) data as a control for each animal, the high-dosage group achieved by the sixth day a performance level not significantly different from that of the low-dosage group. The decrease in response rate seen to result from the change of task on the seventh alcohol treatment day is due to the presentation of the new task, and this decrease is greater with $1.0 \mathrm{~g} / \mathrm{kg}$ alcohol than with $.5 \mathrm{~g} / \mathrm{kg}$ alcohol. Thus, the basic transfer or generalization decrement

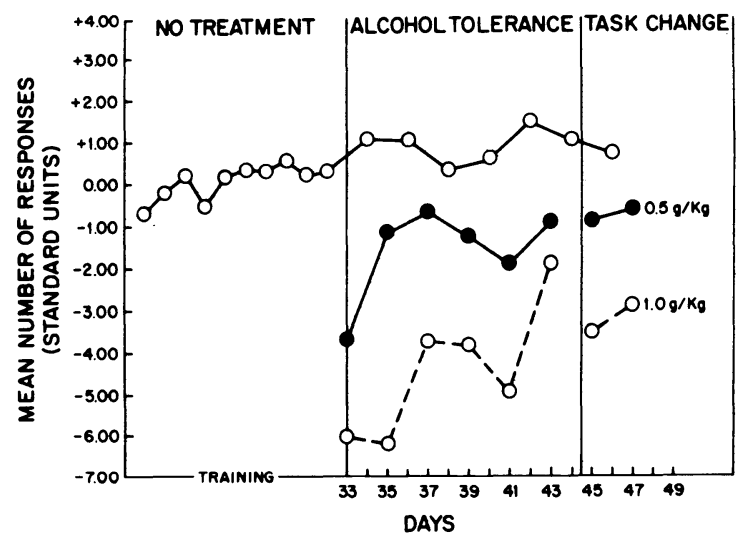

Figure 1. Standard deviation units of barpressing and licking rates in the acquisition (alcohol tolerance) and transfer (task change) phases of Experiment 1. effect was here found to be a function of alcohol dosage, there being less transfer the greater the dosage.

Consistent with previous findings for the treadmill and temporal circular maze, this transfer effect was not found in the case of response duration.

\section{EXPERIMENT 2}

\section{Method}

Subjects. Twenty-four male albino rats were used as subjects. One animal was lost due to a respiratory ailment during the course of the study, and two animals were discarded as a result of procedural error. The results are thus based on 21 animals.

Apparatus. The basic apparatus consisted of four waterdelivery operant boxes as described above.

Alcohol dosages. Alcohol was administered i.p. in a $1.4-\mathrm{g} / \mathrm{kg}$ amount. The injection solution was $23.75 \%(\mathrm{~V} / \mathrm{V})$ of $95 \%$ ethanol and distilled water.

Procedure. All animals were adapted to a $23 \frac{1}{2} 2-\mathrm{h}$ waterdeprivation schedule and maintained on this schedule throughout the experiment. Animals were trained to a stable level of CRF responding in the operant chambers before treatments began.

Rats were assigned randomly to one of three experimental groups: (1) The behavioral group received alcohol injections on treatment days $10 \mathrm{~min}$ prior to the daily 30 -min session. (2) The physiological group received alcohol injections on treatment days immediately after the daily session. (3) The physiological control group received alcohol injections on treatment days $3 \mathrm{~h}$ after the session. To control for drug-related practice during the treatment phase of the experiment, no tests were administered, but were postponed until the number of treatment days was 15 , the point at which the nondrug-related practice tolerance was achieved previously (LeBlance et al., 1973). To control for debilitation due to the water-deprivation schedule imposed on the animals, at least 1 day of normal running was interspersed between the treatment days. The physiological control group was included to assess alcohol effects in the physiological group which might be detrimental to practice effects or learning in the physiological group (e.g., punishment or retrograde amnesia).

The experiment consisted of three phases. Baseline conditions consisted of the initial training and a placebo control inserted after a stable CRF level of responding had been demonstrated. The placebo control day consisted of i.p. injections of isotonic saline $10 \mathrm{~min}$ prior to the operant session for all animals. Treatment was the initiation of alcohol injections for all animals according to grouping. The test phase was made up of alcohol injections for all animals $10 \mathrm{~min}$ prior to the session. As with the treatment phase, a day of normal running was interposed between test days. Baseline lasted 26 days, the placebo control being given on the 26th day. Treatment lasted 38 days; the test phase consisted of a total of 5 days with 3 test days.

\section{Results}

Response rate. The results are summarized in Figures 2 and 3. The behavioral group was composed of eight animals, the physiological group six animals, and the physiological control group seven animals. Due to the unequal number of animals per group, a planned analysis of variance was abandoned in favor of two group comparisons. All related comparisons are sign tests and all independent comparisons are Mann-Whitney $\mathrm{U}$ tests.

The behavioral group's total number of responses for the 30-min session on the first test day (equivalent 


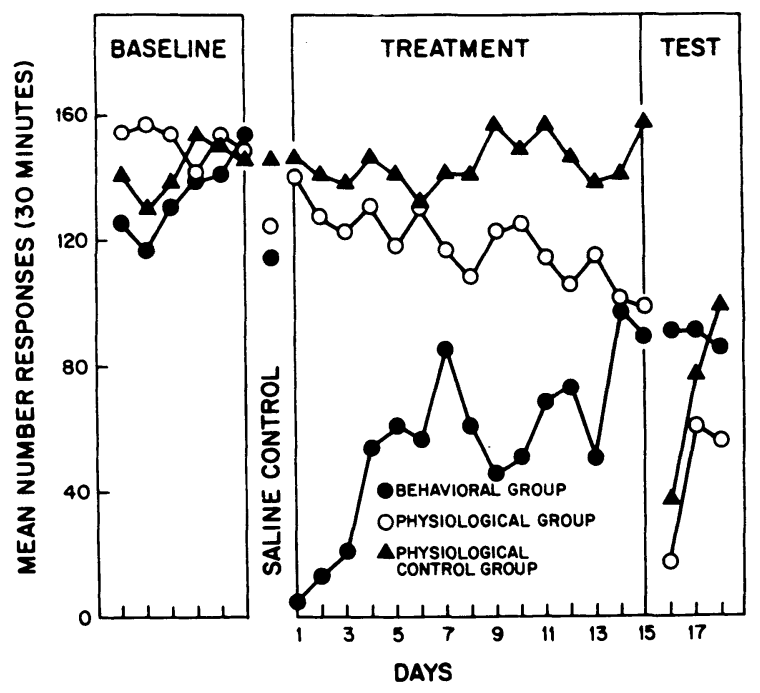

Figure 2. Barpressing rate after presessional alcohol injections (test) as a function of pre- and postsessional injections in tolerance acquisition (treatment) in Experiment 2.

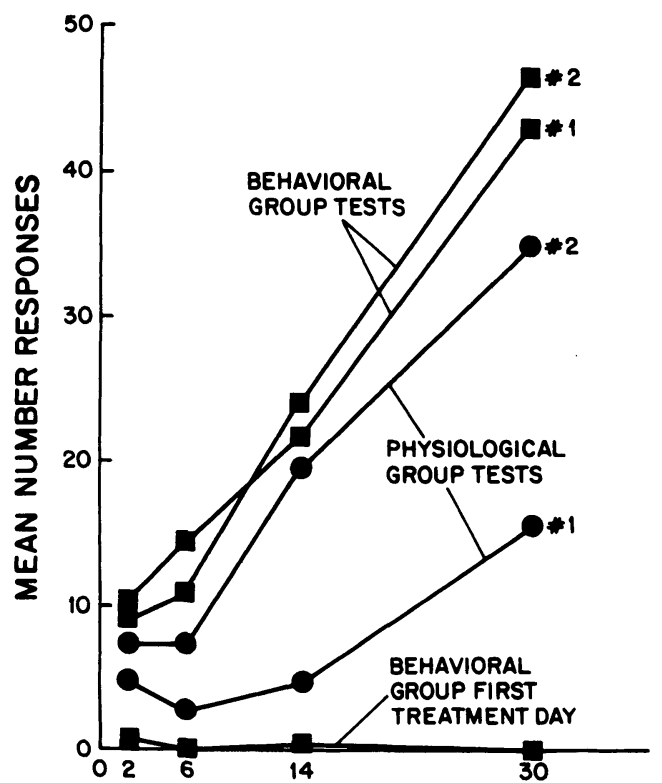

Figure 3. Number of responses within the experimental sessions indicated (see text) for Experiment 2.

to the sixteenth treatment day) was not significantly different from that group's placebo control day $(p=.363)$, while there were significant differences between the physiological group's placebo performance and the first test day $(p=.016)$ as well as the physiological group's placebo and Test Day 1 responses $(p=.008)$. On Test Day 1 the behavioral group differed significantly from the physiological group $(p=.010)$ and from the physiological control group $(p=.036)$, but the physiological groups did not differ from each other $(p=.418)$. Although there is a significant improvement in the combined physiological group's performance from Test Day 1 to Test Day $2(\mathrm{p}=.033)$, and although there is no significant difference between any of the groups on either Test Day 2 or 3, the difference between the placebo day and Test Day 2 as well as the difference between the placebo day and Test Day 3 remains for the combined physiological groups $(\mathrm{p}=.006)$.

Response duration. There is no significant difference in the average time per response between the physiological group's placebo day and Test Day $1(p=.500)$ or between the physiological control group's placebo day and Test Day $1(p=.656)$. There is no difference between the groups on Test Day $1(\mathrm{p}=.114, .496$, $.141)$.

\section{Discussion}

From the results it can be seen that practice within the drug state (behavioral group) vis a vis equal drug experiences without the practice (physiological groups) resulted in a greater facility in operant responding, as reflected by response rate in the initial test situation. By the sixteenth treatment day, tolerance not attributable to learning should have been complete (LeBlanc et al., 1973), but was not in terms of response rate. It can be seen that the learning, when allowed, was very rapid, composing. essentially only 1 test day. For this measure interspersing test days throughout the treatment phase, as was done by LeBlanc et al. (1973), may have masked the learning effect.

That there was a significant tolerance acquisition not attributable to learning may be seen in the difference between the first treatment day for the behavioral group and the first test day of the physiological groups $(p=.020)$. It is, however, impossible to eliminate the attribution of learned tolerance to the physiological groups. For example, the alcohol injection immediately after the operant session (physiological group) appeared to result in a progressive depression of response rate, as a significant difference between the physiological and physiological control groups appears on Treatment Days 14 and 15 $(p=.017)$ as well as on the normal run between these treatment days $(p=.017)$. A conditioned suppression explanation of this effect would cast doubts about ruling out learning for this group.

In summary, on the basis of the present results it is suggested that both transfer of tolerance effects and absolute tolerance level achieved with or without alcohol-related practice are dependent upon the measure of tolerance employed. To the extent that the measure of tolerance is a behavioral measure, and to the extent that different behavioral measures reflect different central nervous activities involved in tolerance, the assessment of tolerance should be dependent upon the measure employed.

\section{REFERENCES}

Chen, C. S. Training schedule in a two-turn task in a temporal circular maze. Psychonomic Science, 1967, 9. 273-274.

Chen, C. S. A study of the alcohol-tolerance effect and an introduction of a new behavioral technique. Psychopharmacologia (Berlin) 1968, 12, 433-440.

Crow, L. T., Westveer, J. J., \& Kass, R. A. Satietydependent microbehaviors in water ingestion by the rat: The effects of alcohol on response duration. Physiological Psychology, 1976, 4, 163-165. 
Kalant, H. Pharmacological and behavioral variables in the development of alcohol tolerance. In Pharmacology and the future of man. Proceedings of the 5th International Congress of Pharmacology (Vol. 1). Basel, Switzerland: Karger, 1973.

Kalant, H., LeBlanc, A. E., \& Gibbins, R. J. Tolerance to and dependence on some non-opiate psychotropic drugs. Pharmacological Revien's, 1971, 23, 135-191.
LeBlanc, A. E., Gibbins, R. J., \& Kalant, H. Behavioral augmentation of tolerance to ethanol in the rat. Psychopharmacologia (Berlin) 1973, 30, 117-122.

(Received for publication February 10, 1977.) 